\title{
Réplique au compte rendu de leur ouvrage intitulé $D u$ comptoir au réseau financier. L'expérience historique du Mouvement Desjardins dans la région du centre du Québec, 1909-1970 (Montréal, Boréal, 1995, 388 p.), par Ronald Rudin, paru dans le volume 49,3 (hiver 1996) : 443-444.
}

\section{Roger Levasseur et Yvan Rousseau}

Volume 50, numéro 1, été 1996

URI : https://id.erudit.org/iderudit/305503ar

DOI : https://doi.org/10.7202/305503ar

Aller au sommaire du numéro

Éditeur(s)

Institut d'histoire de l'Amérique française

ISSN

0035-2357 (imprimé)

1492-1383 (numérique)

Découvrir la revue

Citer ce compte rendu

Levasseur, R. \& Rousseau, Y. (1996). Compte rendu de [Réplique au compte rendu de leur ouvrage intitulé Du comptoir au réseau financier. L'expérience historique du Mouvement Desjardins dans la région du centre du Québec, 1909-1970 (Montréal, Boréal, 1995, 388 p.), par Ronald Rudin, paru dans le volume 49,3 (hiver 1996) : 443-444.] Revue d'histoire de l'Amérique française, 50(1), 132-135. https://doi.org/10.7202/305503ar 
Réplique de Roger Levasseur et Yvan Rousseau au compte rendu de leur ouvrage intitulé $D u$ comptoir au réseau financier. L'expérience historique du Mouvement Desjardins dans la région du centre du Québec, 1909-1970 (Montréal, Boréal, 1995, 388 p.), par Ronald Rudin, paru dans le volume 49,3 (hiver 1996): 443-444.

C'est connu, la communauté historienne n'est pas ce milieu harmonieux que l'on aime se représenter. Les différends entre chercheurs s'expriment habituellement selon un certain décorum et dans le respect de certaines règles communément acceptées par les organismes consacrés à la diffusion de la recherche. Manifestement, Ronald Rudin, dans le compte rendu de notre ouvrage Du comptoir au réseau financier, s'est placé au-dessus de ces règles. En effet, son texte n'est pas un compte rendu; il relève strictement de l'opinion partisane. Il vise à discréditer deux concurrents qui, dans leur livre, proposent de nouvelles interprétations de l'histoire du Mouvement Desjardins et remettent en question des analyses que l'historien de l'Université Concordia avait auparavant menées sur le même sujet. Plutôt que d'amorcer un débat, Rudin a préféré l'esquive et la rumeur en laissant planer des doutes sur l'intégrité des auteurs.

Le procédé utilisé par Rudin est pour le moins disgracieux: il suggère l'idée d'une compromission des auteurs avec les dirigeants des caisses. Pour appuyer son énoncé, Rudin puise toute son inspiration dans la courte préface de Jean Marineau de la Fédération régionale des caisses du centre du Québec et dans notre avant-propos. Qu'y trouve-t-il? Essentiellement trois choses: que Jean Marineau éprouve un «sentiment de fierté» devant l'ouvrage, que les auteurs ont obtenu, en 1988-1990, un «encouragement» par une subvention de démarrage et qu'un comité a été consulté. Ces «preuves» lui suffisent pour établir que l'ouvrage ne pouvait être objectif. Puisqu'il faut répondre, clarifions les choses une fois pour toutes. Mentionnons d'abord que la subvention de démarrage du Mouvement Desjardins ne représente qu'une modeste partie (environ 15\%) de l'ensemble des montants obtenus pour le financement de nos recherches, l'essentiel provenant du CRSH, du Fonds FCAR et de l'UQTR. Deuxièmement, le comité de lecture, auquel fait allusion notre perspicace historien, a été formé à notre demande non pas «pour veiller à ce que l'étude soit suffisamment louangeuse», comme il l'affirme, mais dans le but d'authentifier certains événements ou phénomènes traités dans le volume: les enjeux relatifs à la compensation bancaire, les modalités de l'inspection, l'approbation des placements, les problèmes de liquidités, etc. Quoiqu'en dise Ronald Rudin, les dirigeants des caisses n'ont eu absolument rien à voir avec le contenu, la structure ou la forme de l'ouvrage. Celui-ci n'est donc pas une commandite, n'a aucun caractère «officiel» et n'a pas non plus été réalisé pour les fins du $75^{\mathrm{e}}$ anniversaire de la Fédération régionale. Cette dernière a profité de sa publication pour en faire un événement dans le cadre de cet anniversaire.

Une autre «preuve» de cette compromission des auteurs avec les dirigeants des caisses, conclut Rudin, résiderait dans l'usage de pseudonymes dans une section de chapitre du volume. Ici, le ridicule atteint son point culminant. Ce 
«fait curieux», faut-il le rappeler à notre éminent historien, est un procédé couramment utilisé dans les études de milieu, notamment dans les études sociologiques. Ainsi, Everett C. Hughes, dans son volume Rencontre de deux mondes, parle de Cantonville plutôt que de Drummondville. La caisse gérée par Omer B...., c'est effectivement la caisse d'Omer Beaumier et, pendant que nous y sommes, avec le discernement que nous lui connaissons, Rudin aura sans doute remarqué que la caisse de Robert D...., c'est la caisse gérée par Robert Durand de Trois-Rivières. Ce procédé permet d'éviter de singulariser les événements, les lieux et les individus pour mettre l'accent sur les phénomènes sociaux. À tout prendre, les dirigeants du Mouvement auraient sans doute préféré que les individus soient davantage mis en évidence.

Fort d'avoir «dévoilé» cette compromission des auteurs avec les dirigeants des caisses, Rudin présente l'ouvrage comme étant complaisant, sans perspective «analytique» et sans fondement méthodologique rigoureux. À aucun moment toutefois, il ne prend appui sur le contenu du livre pour étayer son affirmation. Loin de l'éloge, le portrait général qui se dégage des analyses présentées dans ce livre est celui d'un mouvement polycentrique, marqué par de profondes disparités de développement et travaillé par de multiples foyers de tensions entre classes sociales, entre l'autonomie des unités de base du Mouvement Desjardins et la centralisation, entre les cadres, les employés, les élus et les membres, entre la ville et la campagne, entre régions. Au total, il s'agit d'une problématique sans doute plus complexe que le modèle simplificateur fondé sur l'opposition entre «pauvres», "petite bourgeoisie traditionnelle» et «nouvelle classe moyenne» qui est à la base des interprétations de Rudin dans son livre (In Whose Interest?, 1990), un livre auquel nous avions nous-mêmes accordé certains mérites dans le contexte de la pauvreté de l'historiographie à l'époque de sa parution $(R H A F, 44,3$, hiver 1991). Dans Du comptoir au réseau financier, nous nous sommes éloignés des thèses de Rudin parce que, selon nous, son approche souffrait d'importantes lacunes tant d'un point de vue théorique que méthodologique. Donnons quelques brèves indications des divergences entre les deux thèses en présence que nous souhaiterions prolonger dans un véritable débat.

L'une des divergences importantes entre les deux ouvrages renvoie au phénomène de la centralisation et, plus largement, à celui du changement au sein du Mouvement Desjardins. Dans la vision linéaire privilégiée par Rudin, tout s'articule autour d'énoncés dichotomiques simples à la manière dont il les affectionne. Pour lui, les changements au sein des caisses populaires sont introduits selon une logique qui part du haut de l'organisation pour se diffuser vers le bas, ce qui l'amène à escamoter complètement le poids des corps régionaux et des grosses caisses dans sa lecture du Mouvement Desjardins. Notre approche tient compte, non seulement des dynamismes du centre, mais accorde aussi un rôle de premier plan à l'action et aux pressions issues de la base du Mouvement Desjardins dans l'analyse des problèmes relatifs au changement.

Dans notre livre, nous avons également pris nos distances à l'égard de la lecture de premier niveau que fait Rudin des discours tenus par les divers 
protagonistes du Mouvement Desjardins, parce qu'elle relevait davantage de l'histoire spontanée que d'une démarche historique rigoureuse. Ainsi en estil, par exemple, de sa confusion entre la notion de «classes laborieuses» utilisée par les promoteurs du Mouvement (qui, dans leur langage, renvoie à l'univers de la petite bourgeoisie et des petits producteurs) et celle de poors dont Rudin se sert pour désigner les plus démunis. Le respect d'un principe épistémologique élémentaire, à savoir la distance critique par rapport au langage commun, aurait permis au professeur de Concordia d'éviter pareille confusion. Or, une bonne partie de son interprétation du Mouvement part de cette méprise. Cette lecture primaire des discours amène Rudin à s'enfermer dans une conception peu dynamique des rapports entre tradition et modernité. Pour lui, le projet des premiers animateurs des caisses Desjardins est essentiellement une tentative de recréer la société québécoise du XIX $\mathrm{X}^{\mathrm{e}}$ siècle et, conséquemment, de rétablir le pouvoir des élites locales. Sur cet aspect des choses, nous faisons un bout de chemin avec Rudin, mais ce qu'il n'a pas saisi dans ce projet, c'est qu'il comporte un autre volet tout aussi important: celui de la réinsertion de la petite bourgeoisie et de la paysannerie dans l'économie de marché. Il s'agit d'une manière particulière pour la société québécoise de l'époque de définir son rapport à la modernité.

Nous nous sommes enfin éloignés des thèses soutenues par Rudin sur la question des rapports entre les caisses et les classes sociales. Tout en reconnaissant le rôle capital assumé par la petite bourgeoisie au sein des caisses, notre ouvrage met également l'accent sur la place non moins importante qu'y occupe la paysannerie jusqu'à la Seconde Guerre mondiale, un phénomène qui échappe complètement à Ronald Rudin. Son approche des classes comporte plusieurs déficiences sur le plan méthodologique. Ainsi, dans sa démarche empirique, il utilise trois méthodologies distinctes de façon à soutenir les besoins de sa thèse: 1) l'analyse de discours lorsqu'il s'agit de retracer les agents de la nouvelle classe moyenne (en prenant soin de mettre en valeur ce qui, dans les discours des protagonistes, s'accorde avec son hypothèse de départ et d'évacuer ce qui la contredit...); 2) le classement socioprofessionnel quand il veut cerner le poids de la petite bourgeoisie dans les deux caisses urbaines qui lui servent d'échantillon; 3) des strates établies en fonction de la valeur des propriétés immobilières lorsqu'il aborde la situation dans trois caisses rurales. L'usage qu'il fait du concept de nouvelle classe moyenne et des antagonismes entre cette dernière et la petite bourgeoisie «traditionnelle» est tout aussi problématique. Sa reconstruction historique des «conflits de classes» au sein du Mouvement tient, dans une large mesure, à l'équation réductrice qu'il effectue en associant, d'un côté, la petite bourgeoisie aux deux notaires montréalais, Wilfrid Guérin et Eugène Poirier, et, de l'autre, les agents de la nouvelle classe moyenne au sénateur Vaillancourt. Par ailleurs, son concept de nouvelle classe moyenne n'est nulle part défini de façon opératoire. Avec une telle rigueur, on comprend que Rudin ait complètement occulté la masse des paysans qui vont fournir au Mouvement l'essentiel de ses dirigeants au cours de l'entre-deux-guerres. Dans son ouvrage, en effet, la paysannerie se trouvera assimilée tantôt aux «pauvres», tantôt à la petite bourgeoisie. Encore une fois, tout dans son récit 
gravite autour des besoins de sa thèse et ceci, au mépris des règles heuristiques les plus élémentaires.

Les remarques méthodologiques de Rudin concernant la rigueur de notre approche ne méritent pas que l'on s'y attarde longuement: elles sont du même ordre que les autres. Ce que Rudin ne dit pas, c'est que notre étude s'appuie principalement sur des données sérielles relatives à la totalité des caisses de la région et qu'il s'agit, dans la plupart des cas, de données originales. Ce qu'il oublie également de mentionner, c'est que l'échantillon utilisé pour approfondir certaines questions comporte pas moins de 60 caisses, qu'il représente près de la moitié des caisses en activité dans la région pendant l'après-guerre et qu'il a permis de cerner des dimensions particulières du fonctionnement des coopératives. Ce groupe témoin, comme nous l'avons précisé dans l'appendice méthodologique, est représentatif de la diversité régionale (milieux urbains et ruraux, rive nord et rive sud, grosses caisses et petites caisses, comtés d'appartenance), mais s'écarte de la moyenne, selon le critère de l'âge et de l'actif. La critique méthodologique est pour le moins étonnante de la part d'un historien qui, à partir de cinq caisses populaires (dont il n'a jamais discuté la représentativité), procède à des généralisations pour l'ensemble des caisses au Québec. Encore plus étonnant pour un historien qui, à partir des discours tenus par un sénateur, deux notaires et un chef inspecteur pendant les années 1940, conclut à la domination de la nouvelle classe moyenne sur l'organisation du Mouvement Desjardins. Que dire enfin de son analyse des politiques de prêts qui, elle, ne s'appuie que sur la seule Caisse de Lévis, un établissement dont la taille ne trouve aucun équivalent parmi les caisses populaires de l'époque étudiée.

En définitive, Rudin a préféré éviter de confronter nos interprétations aux siennes. Si son compte rendu n'apprend rien au lecteur sur notre ouvrage, il poursuit sans doute d'autres visées qui ne relèvent pas de la démarche historique dont il se réclame. Nous avons cru utile de faire cette mise au point pour qu'enfin ait lieu un véritable débat sur l'histoire des caisses au Québec. 